\title{
Cross-Layer Multipath Multichannel MAC protocol for MANETs
}

\author{
Nawaf S. Mirza*, Hamish Taylor*, Mohamed A. Abdelshafy*, Peter J. B. King*, Imed Romdhani ${ }^{\dagger}$, Atif A. Alghamdi * \\ ${ }^{*}$ School of Mathematical and Computer Sciences \\ Heriot-Watt University, Edinburgh, UK \\ \{nm246, h.taylor, m.abdelshafy, P.J.B.King, aaa148\}@hw.ac.uk \\ ${ }^{\dagger}$ School of Computing, Edinburgh Napier University, Edinburgh, UK
}

\{I.Romdhani\}@napier.ac.uk

\begin{abstract}
Utilising multiple disjoint paths in multiple channels can improve network performance by enabling a node to reroute data along discovered paths seamlessly when link failure is detected. However, depending on a stale/invalid route to recover from a broken link could increase the delay to recover from the broken link and degrade the network performance. In this paper, we propose a new MAC protocol (RIVC-MB) to enhance communication reliability in the multipath multichannel routing protocol. The reliability of transmitting/re-routing the data packet in multipath multichannel routing protocol is improved by providing early route invalidity detection and early switchover. Waiting time to access the medium is also improved, when a node is attempting to access a busy medium, by re-routing the data packet via the alternative route. The RIVC-MB protocol uses the cross-layer interaction between MAC and routing protocols to achieve these goals. The proposed protocol is implemented and extensively evaluated using the NS-2 simulator. Simulation results show that the new proposed protocol improves the endto-end delay, and reduces both the number of route error control packets and the number of dropped data packets in the routing layer. It also reduces the collision rate in the MAC layer in a dense network.
\end{abstract}

Keywords- Route validity detection, Multipath Multichannel routing, Reliability, IEEE 802.11, Backoff, Switchover.

\section{INTRODUCTION}

A Mobile Ad-hoc Network (MANET) is a collection of mobile nodes which form a self-configurable and selforganising network without relying on any fixed infrastructure. Mobile nodes in MANET communicate directly, if they are in the same transmission range, while those which are not can use other nodes to relay their packets in a multi-hop manner. Design a routing protocol for MANETs is challenging due to the dynamic topology of mobile nodes and unique characteristics of the wireless channels.

Several protocols to improve routing protocols in MANETs have been proposed. Multipath routing appears to be one of the more promising solutions to design a robust routing protocol for them. In a multipath routing protocol, more than one path from the source to the destination node is discovered. When a path gets broken due to topology change or node failure, the next path (alternative) is used to recover from the broken link without initiating another route discovery. The discovered paths can be node-disjoint (no nodes in common) or linkdisjoint (no links in common). Node-disjoint alternative paths make the routing scheme more robust and offer a higher degree of fault-tolerance as node failures do not affect the other paths. Most of the proposed multipath routing protocols [1], [2] employ a single shared medium of communication and due to the broadcast nature of radio communication, the level of inter-path interference [3] is pronounced even if the node-disjoint property is satisfied. This problem is also known as the route-coupling [4] where simultaneous communications occur in multiple paths which are physically located in the interference range of each other.

Utilising multiple non-overlapping frequency channels, which is already permitted in the IEEE 802.11 standard [5], can help overcome this issue and improve network performance by discovering multiple paths in multiple channels. Hence, a multipath multichannel routing protocol [6], [7] can be considered as a channel-disjoint routing protocol which can help to mitigate inter-path interference problems among multiple discovered paths. However, depending on a stale/invalid route at the receiver/alternative node to recover from a broken link, can degrade network performance and increase the delay to recover from the broken link.

Motivated by this, we propose a new mechanism called Route Information Validity Check (RIVC) which aims to mitigate the issue of transmitting/re-routing the data packet to a node which has stale (invalid) route information towards the final destination. The optional access mode Request-ToSend/Clear-To-Send (RTS/CTS) in IEEE 802.11 DCF has been modified to enable the transmitter node to check the route information validity at the receiver node while exchanging RTS/CTS frames. In the case where the receiver node has invalid routing information, then the transmitter node should invoke an alternative path which is in a different channel. This will allow early detection of a stale/invalid route and an early switchover.

In IEEE 802.11 DCF, the absence of the corresponding acknowledgement frame (CTS/ACK) for the transmitted frame (RTS/DATA) signals a collision with another transmission and that the intended receiver has possibly not received the frame. Hence, a transmitter should initiate the error recovery process by retransmitting the failed frame after observing the backoff rules [5]. The transmitter should retry transmitting the frame until the transmission is successful, or until the relevant retry limit is reached, whichever occurs first. However, repeating retransmission in the MAC protocol can increase contention/collision in a busy medium and hence cause conges- 
tion in the channel. In a multipath multichannel network, when a broken link is detected, the transmitter can try to recover from it via the alternative path using the other channel.

This motivated us to introduce a Modified Backoff (MB) mechanism. The MB mechanism enables the transmitter node to invoke an alternative path in the alternative channel, if available when the Contention Window $(\mathrm{CW})$ reaches pre-set threshold criteria. This would help to reduce the waiting time to access a busy medium. Additionally, it would help to reduce inter-path interference and collisions in the channel.

In this paper, we propose a new MAC protocol called (RIVC-MB) which consists of two mechanisms RVIC and MB. It aims to improve the performance and the reliability of communication in the multipath multichannel routing protocol. The first mechanism is RIVC which aims to forecast invalid route information at the receiver node and provide an early switchover to an alternative path, if possible at the MAC layer level. The second mechanism is $\mathrm{MB}$ which aims to reduce the waiting time for a node which is attempting to access a busy medium by re-routing the data along the alternative path when the CW reaches pre-set threshold criteria. Both mechanisms utilise cross-layer interaction between MAC and the routing layer to check route information validity and provide an alternative node to communicate with at the MAC layer level.

The proposed protocol could operate with many types of multipath multichannel routing protocols to improve communication reliability. To proof the concept of the proposed protocol, we used the Receiver Directed Transmission (RDTMAC) protocol [8] with a single radio interface to implement the multi-channel MAC protocol and the Reliable Multi link failure Route Migration over Multiple Channel (RMMMC) [9] routing protocol as a means to implement the multipath multichannel routing protocol. However, the proposed protocol is not limited to these means.

The rest of this paper is organized as follows. Section II summarises related works. Section III identifies the problems to be addressed. Section IV proposes our protocol. In Section V presents the simulation parameters, while Section VI presents the performance results. Section VII summarizes key results and issues.

\section{RELATED WORK}

In this section, we briefly review some related work which is similar to this work.

Campbell et al. [10], proposed a Multi-Channel MAC (MC-MAC) solution to reduce the waiting time when a node attempts to access a busy medium in Wireless Sensor Network. MC-MAC modified the original backoff algorithm of IEEE 802.11 DCF to invoke channel switching based on a set of threshold criteria (after three access retries). When the threshold is reached, the node should switch to another channel and sense if the medium is busy. If so, it should switch to yet another channel. If a channel medium is free, the node may transmit the packet. If all channels are busy, the transmitter should revert to a random backoff timer. MC-MAC considers only a single hop network scenario where the transmitter node sends the data packet to the sink.
Mirza et al. [8] proposed a routing RDT-AODV and MAC protocol RDT-MAC (which based on IEEE 802.11 DCF) to utilise multiple channels in ad-hoc wireless networks based on the Receiver-Directed Transmission communication scheme [11]. In an RDT-MAC setup, each node is equipped with a single half-duplex transceiver. RDT-MAC facilitates channel assignment and negotiation to agree on a communication channel without using a common control channel or needing tight time synchronisation. At network initialisation, each node is assigned a home channel to listen to when not transmitting, using the following equation [8]:

$$
\left(H \_C h=N_{-} I d \bmod T \_C h\right)(1)
$$

where $H_{-} C h$ is a node's home channel, $N_{-} I d$ is the node's IP address and $T \_C h$ is the total number of available channels in the network. The home channel for any node in the network can be calculated using equation (1). In the RDTAODV protocol, route discovery and maintenance are carried out in the destination node's home channel. Although RDTMAC facilitates channel assignment and negotiation to utilise multiple channels in the network, it does not provide flexibility as it assigns channels deterministically and may cause network partitioning.

Mirza et al. [9] proposed a Reliable Multi link failure Route Migration over Multiple Channel (RMMMC) routing protocol. RMMMC is a multipath multichannel routing protocol which aims to improve route discovery in RDT-AODV and overcome its issues. Additionally, RMMMC utilises a new route recovery mechanism which is based on the discovered multipath in multiple channels. In the RMMMC protocol, multiple route discovery processes are initiated in multiple channels for each destination. This enables source nodes to discover multiple node, link and channel-disjoint paths in multiple distinct channels. For simplification, however, RMMMC limits the number of route discovery processes to two per destination to reduce the routing packets overhead in the network, to reduce the amount of channel switching and to simplify implementation of the algorithm.

The RMMMC routing protocol supports route accumulation in the route discovery process (RREQ/RREP). Hence, each source node will be aware of the full multi-hop route in different channels. Intermediate nodes involved in the routing path will be informed about the full multi-hop path in the alternative channel and updates their alternative table. When a node detects a link failure, it tries to recover from the broken link by using the alternative path. If an alternative route is available, then the node calculates the alternative node's home channel using equation (1) and switches its channel to re-route the data packet along the alternative path. Otherwise, the node drops the data packet and notifies its upper layer which will send RERR to the source node. Although RMMMC improves the performance network performance and provides a multihop channel-disjoint alternative route, it does not consider the validity of the alternative route which may cause a node to reroute data to an alternative with stale route.

\section{PRoblem StATEMENT}

Utilising multiple paths in multiple channels can help to recover from a broken link faster and reduce congestion/interference in a busy channel by re-routing data to a 
pre-discovered route in a different channel. However, a node is equipped with only a single transceiver and will be aware of the activities that occur in the channel that it listens to. The transmitter may transmit/re-route data packet to a nexthop node which has invalid routing information. This may cause the data packet to be dropped and increase the delay to recover from the broken link.

Although RMMMC discovers two paths in different channels to each destination, it only uses one path as the main path for data transmission and only relies on the second path as an alternative when link failure is detected. Furthermore, since a data packet is only transmitted on one path, the validity of the cached route may become stale or not reflect the current state of the network topology. Hence, the transmitter may try to transmit/re-route a data packet to a next-hop node which has an invalid route.

Receiving a data packet while the node has invalid routing information toward the destination node has consequences; it may unnecessarily cause neighbour nodes to backoff from contending on the medium and delay sending their data, with no gains to the network. Furthermore, the medium is saddled with data packets which have invalid routes and may waste node and network resources thereby.

In IEEE 802.11 DCF, a transmitter should retry transmitting a failed frame until it is successful, or until the relevant retry limit is reached. However, repeatedly retransmitting in a busy medium can increase contention/collisions and cause congestion in the channel.

This paper aims to address the issues mentioned above and improve the reliability of communication in a multipath multichannel routing protocol.

\section{PRoposed PRotocol}

The proposed protocol RIVC-MB is designed to improve the reliability of data packet transmitting/re-routing and to reduce the waiting time when a node is attempting to access a busy medium in a multipath multichannel routing protocol. It specifies two mechanisms to improve the reliability of communication and to reduce the waiting time to access a busy medium in a multipath multichannel wireless ad-hoc network.

The first mechanism is a modification to the optional access mode (RTS/CTS) in IEEE 802.11 DCF called Route Information Validity Check (RIVC). It aims to mitigate the issue of transmitting/re-routing the data packet to a node which has invalid route information toward the final destination during an RTS/CTS exchange. If routing information at the receiver node is stale, then the transmitter performs an early switchover and re-routes the data via an alternative route.

The second mechanism is a modification to the backoff and error recovery mechanism in IEEE 802.11 DCF called Modified Backoff (MB) mechanism. It aims to reduce the waiting time when a node is attempting to access a busy medium by re-routing the data via an alternative route if available when the Contention Window $(\mathrm{CW})$ reaches pre-set threshold criteria.

The proposed protocol provides the following benefits to the network:
- Provide early route invalidity detection and early switchover.

- Utilise the existing RTS/CTS with a slight modification to check route validity at the intermediate nodes toward the final destination.

- Improve the reliability of data transmission via to an alternative route in a different channel, if available.

- $\quad$ Reduce the waiting time to access a busy medium by invoking an alternative route when the $\mathrm{CW}$ reaches a pre-set threshold.

- Reduce the risk of transmitting/re-routing a data packet, which has an invalid route at the receiver node, to occupy the medium and waste node and network resources.

The proposed mechanisms are presented in detail in the following sections.

\section{A. MAC Control Frames}

IEEE 802.11 DCF defines the control frames RTS/CTS to reserve the medium, alleviate the hidden node problem and improve the reliability of communication in the wireless medium. Furthermore, they provide faster collision inference and validation of the transmission path existence [5].

In order to support such functionality for the RIVC mechanism, slight modifications to the RTS and CTS frames format are introduced.

RTS frame: In the RTS frame, one field is added as shown in Figure 1:

- Final_Destination_Address: This is the address of the final destination node of the data packet. Including this field enables the receiver of the RTS frame (intermediate node) to check the validity of its route information towards the final destination.

\begin{tabular}{l|c|c|c|c|c|} 
Bytes 2 & \multicolumn{2}{c}{4} & 6 & 6 & 6 \\
\hline Frame control & $\begin{array}{c}\text { Destination } \\
\text { ID }\end{array}$ & $\begin{array}{c}\text { Address 1 } \\
\text { Receiver } \\
\text { address }\end{array}$ & $\begin{array}{c}\text { Address 2 } \\
\text { Transmitter } \\
\text { address }\end{array}$ & $\begin{array}{c}\text { Address 3 Final } \\
\text { destination address }\end{array}$ & FCS \\
\hline
\end{tabular}

Fig. 1: New RTS frame Structure.

CTS frame: In the CTS frame, one field is added as shown in Figure 2:

- Validity_flag: This indicates the validity of the routing information at the receiver node. Based on this flag, the transmitter node can decide to continue transmitting the data packet or to invoke the alternative route, if possible. Furthermore, the validity flag in the CTS frame will help neighbour nodes, which hear this CTS, decide whether to backoff from contending within the medium or not. 


Bytes 2
\begin{tabular}{|c|c|c|c|c|}
\hline Frame control & Destination ID & Address 1 Receiver address & $\begin{array}{c}\text { Validity } \\
\text { flag }\end{array}$ & FCS \\
\hline
\end{tabular}

Fig. 2: New CTS frame Structure.

\section{B. Cross-Layer interaction between MAC and routing layers:}

The proposed protocol utilises cross-layer interaction between the MAC and routing layers to provide the aforementioned functionality. We implement a new extension to the Network Simulator version 2 (NS-2) [12] to enable cross-layer interaction between the $\mathrm{MAC}$ and the routing protocols. In the proposed protocol, the MAC protocol interacts (consults) with the routing protocol in the following three cases: the first case, when the receiver node receives an RTS, it checks its route information validity in the routing table towards the final destination before sending CTS back. The second case, when the transmitter receives CTS with an invalid flag, it checks for an alternative node from its alternative table in the routing layer. And finally, when the $\mathrm{CW}$ in the MAC protocol reaches a pre-defined threshold, the transmitter node consults its alternative table in the routing layer for an alternative route in a different channel. For simplicity, we assume that the MAC address is the same as the IP address which is also assumed in the NS-2 implementation, see section 14.6 in [13].

The proposed protocol in the MAC layer is not intended as a substitute for a network layer routing protocol. Routing protocols are still required to find and maintain the multiple paths. However, supporting routing protocols in mobile networks with the proposed MAC mechanisms should improve transmitting/re-routing reliability in a multipath multichannel routing protocol. Additionally, they can reduce the collision and wait time to access a busy medium, and improve end-toend performance.

\section{Route Information Validity Check (RIVC) mechanism}

The RIVC mechanism adds an extra check (which utilises a cross-layer interaction with the routing layer) while the transmitter and the receiver are exchanging modified RTS/CTS frames. It aims to improve the use of the medium by enabling only a data packet with valid routing information at the receiver node to be transmitted and consume bandwidth on the medium. This check is important as only data packets with valid routing information should be processed in the routing protocol and forwarded to their final destination. Furthermore, RIVC enables the transmitter node to be informed about the invalidity of the route at the receiver node, to consult its routing layer and re-route the data packets along an alternative route, if available. This should provide faster route invalidity detection and an early re-routing process without incurring extra overhead or delay. Additionally, it should reduce the number of dropped data packets due to invalid route information which unduly consumes bandwidth on the medium.

The process of handling the modified RTS/CTS frames in RIVC at the transmitter, receiver and neighbour nodes is as follows:
1) The transmitter node: Switches its interface to the receiver node's home channel and transmits the modified RTS frame which includes the final destination address (address number 3 ) after observing the medium access rules.

2) The receiver node: When the receiver node receives the RTS frame, it checks its routing table (cross-layer interaction) to determine the validity of its routing information towards the final destination (address 3 in the RTS frame). If it has valid routing information, then the receiver node sends CTS with a valid flag which signifies the validity of its routing information towards the final destination. On the other hand, if its routing information towards the final destination is invalid, then the receiver node sends CTS with an invalid flag.

3) The transmitter node: When the transmitter node receives the CTS frame, it checks the validity flag. If the flag is valid which signifies that the receiver node has valid routing information towards the final destination, then the transmitter node sends the data frame as in the IEEE 802.11 DCF which is illustrated in Figure 3. On the other hand, if the flag in CTS is invalid, then the transmitter node can conclude that the route in the next hop is invalid and, hence, try to reroute the data packet via its alternative route, if available.

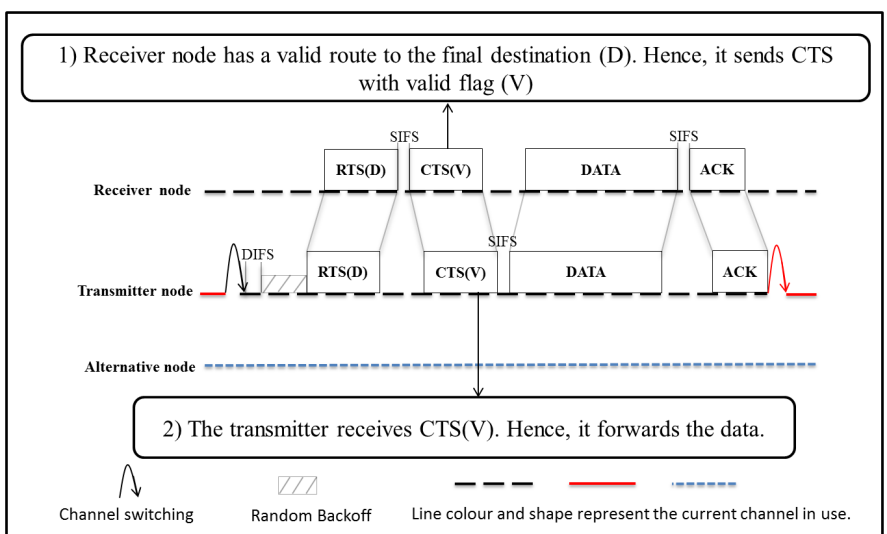

Fig. 3: RTS/CTS exchange in RIVC. Case: the receiver has valid route information.

In this case, the transmitter checks its alternative table in the routing layer (cross-layer interaction) to see if it has a valid alternative node in the alternative channel. If the transmitter does not have an alternative route, then it drops the data packet and notifies the upper layer as in Figure 4. On the other hand, if the transmitter has a valid alternative node, then the transmitter updates the receiver field in the RTS (Address 1) and in a data frame, calculates the alternative node's home channel using equation (1), switches its interface to it, resets the $\mathrm{CW}$ value to the minimum (CWmin) as it is contending/operating on a new channel and then exchanges the RTS/CTS frames.

If the new receiver (alternative node) has a valid 
route towards the final destination (sent CTS with valid flag), then the transmitter transmits the data packet to the alternative node as shown in Figure 5. On the other hand, if the receiver has an invalid route toward the final destination, then the transmitter receives a CTS with an invalid flag. In this case, the transmitter node can conclude that all its available routes (primary and alternative) are invalid and hence drop the data packet and notify the upper layer (routing layer) to send a route error (RERR) packet to the source as illustrated in Figure 6.

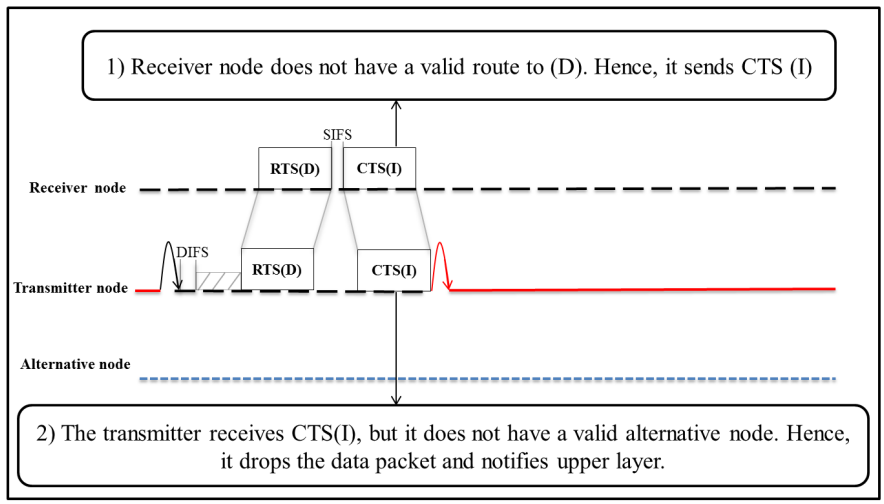

Fig. 4: RTS/CTS exchange in RIVC. Case: receiver and alternative nodes have invalid route information.

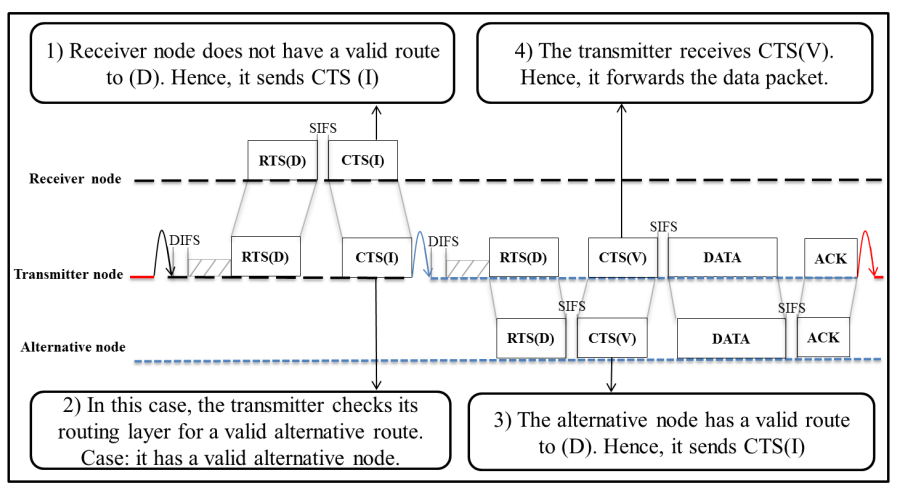

Fig. 5: RTS/CTS exchange in RIVC. Case: receiver has invalid route information and the transmitter has a valid alternative route with valid route information.

4) The neighbour nodes: When neighbour nodes hear RTS frame, they should backoff from contending on the medium and should set their NAV timer. However, in the case of the neighbour nodes hearing a CTS frame, they should act according to the validity flag. If the flag in the CTS is valid, then neighbour nodes should backoff from contending on the medium and set their NAV timer. On the other hand, if the flag in the CTS is invalid, then neighbour nodes should not backoff from contending on the medium as no further communication will follow from the transmitter node.

Note that the RIVC mechanism only seeks to assure that the next-hop node still has valid routing information towards

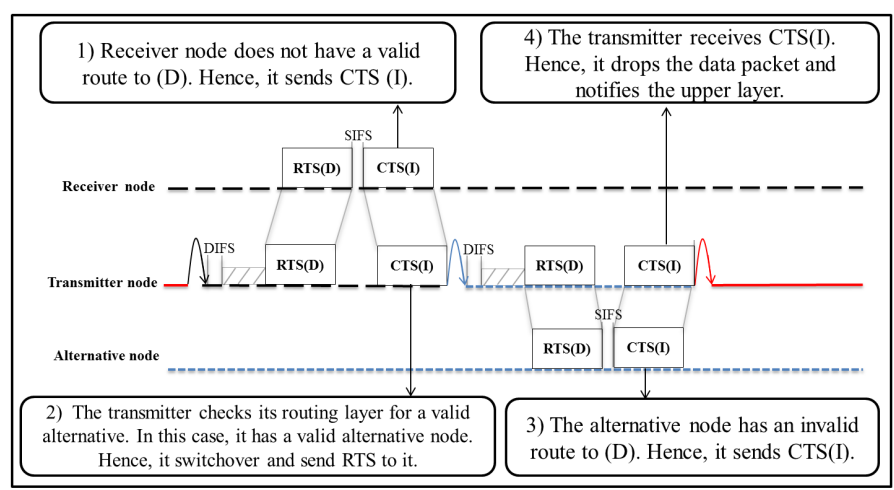

Fig. 6: RTS/CTS exchange in RIVC. Case: receiver has invalid route information, and the transmitter does not have a valid alternative route.

the final destination. However, it does not guarantee that the link in the next 2-hops still exists, as it is difficult and costly to track link connectivity of mobile nodes in a decentralised network, such as a MANET.

\section{Modified Backoff (MB) mechanism}

The MB mechanism modifies the backoff procedure to invoke the alternative route, if available when the $\mathrm{CW}$ value has reached pre-defined threshold criteria. We call this threshold a Channel retransmission Retry Limit (CRL). The CRL threshold is based on the contention window value and will be reached after three retransmissions follow the initial transmission.

When a node tries to transmit and senses the medium is busy or when it transmits a frame and does not receive a corresponding acknowledgement frame, it increments the $\mathrm{CW}$ value and the Short Retry Limit Counter (SRLC) and transmits again. If the $\mathrm{CW}$ value is less than the CRL threshold, then the transmitter node continues its attempts to transmit the frame in the current channel as in the IEEE 802.11 standard. On the other hand, if the CW value has exceeded the CRL threshold, then the transmitter node checks its alternative table in the routing layer to determine if it has a valid alternative route. If it does not have an alternative route, then the transmitter continues the backoff procedure in the current channel as in the IEEE 802.11 DCF protocol. On the other hand, if the transmitter has a valid alternative, then the transmitter updates the destination address field in the RTS (Address 1) and in the data frame to the new receiver address (alternative node), calculates the alternative node's home channel using equation (1) and switches its interface to it. Also, the transmitter resets the $\mathrm{CW}$ value to the minimum (CWmin) as it is contending/operating on a new channel and then exchanges RTS/CTS frames according to the RIVC mechanism in the new channel. Note that the transmitter node should not reset the SRLC and should not change the address of the data packet's final destination.

If the new receiver (alternative node) has valid routing information towards the final destination, then it should send CTS with a valid flag. Then the transmitter node will re-route the data to this node as shown in Figure 7. On the other hand, if the receiver node (alternative node) has invalid routing 
information, then it should send CTS with an invalid flag. In this case, the transmitter node should drop the data packet and notify the upper layer as shown in Figure 8.

Enabling the transmitter node to invoke the alternative route following repeated unsuccessful transmission reduces contention/collisions in the primary channel and also reduces the waiting time to access a busy medium. Furthermore, using RIVC along with the MB mechanism ensures route validity at the alternative node. Hence, the waiting time to access a busy medium should be reduced and fewer data packets with invalid routing information will clog the medium.

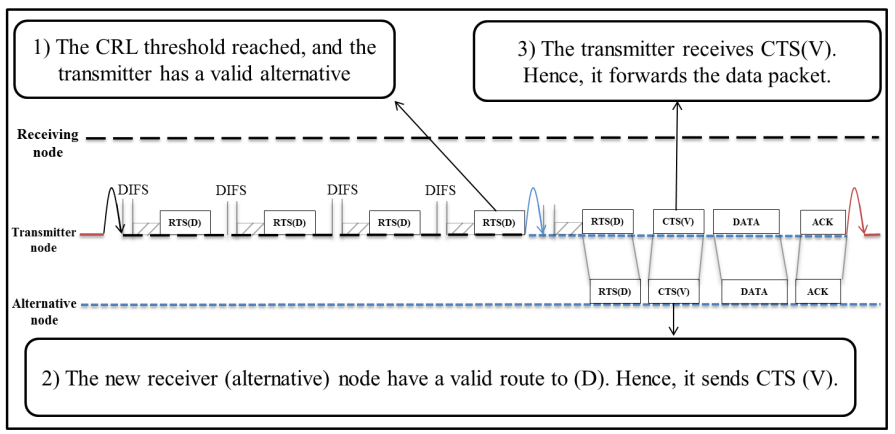

Fig. 7: Contention window in MB. Case: the transmitter has an alternative route with valid route information.

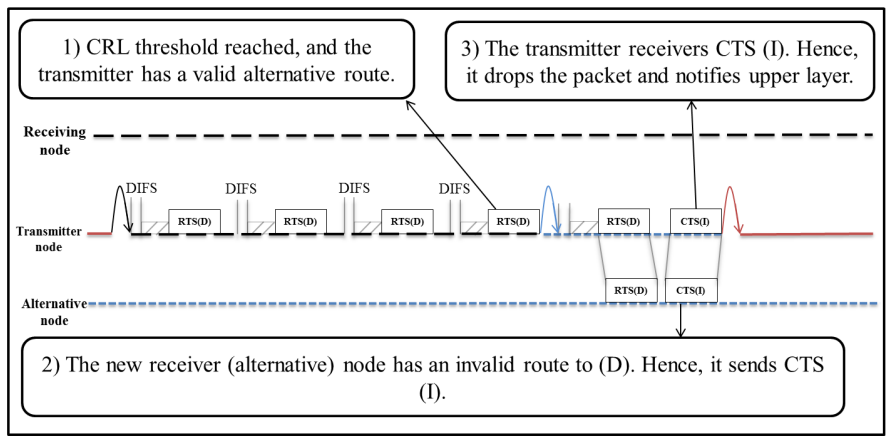

Fig. 8: Contention window in MB. Case: the transmitter has an alternative route, but the alternative node has invalid route information.

\section{Simulation PARAMETERS}

In order to demonstrate the effectiveness of the RIVCMB protocol, the proposed protocol was evaluated and compared in performance to the standard AODV [14] (single path single channel), the RDT-AODV (single path multichannel) and the RMMMC (multipath multichannel) routing protocols. The RIVC-MB protocol was implemented using the NS2.35 simulator [12]. Table I shows the simulation parameters used. Simulation time was 600 seconds. The common default parameters in NS-2 for radio power and threshold levels were adopted. Source-destination pairs were selected randomly. Three orthogonal channels were used for the multichannel protocols. Random way-point was used for the mobility model. Each data point in the figures was an average of 25 runs, with a randomly generated topology. Generally, the $95 \%$ confidence interval was small compared with the value that is reported.
TABLE I: SIMULATION PARAMETERS

\begin{tabular}{|c|c|}
\hline Simulation area & $1000 \mathrm{~m} \times 1000 \mathrm{~m}$ \\
\hline Propagation model & Two-ray ground \\
\hline Traffic type & CBR \\
\hline Packet size & 1000 (bytes) \\
\hline Number of nodes & $50,75,100, \ldots, 225,250$ \\
\hline Number of connections & 50 \\
\hline Max speed & $10(\mathrm{~m} / \mathrm{s})$ \\
\hline Pause time & $20(\mathrm{sec})$ \\
\hline Generation rate & $2(\mathrm{packet} / \mathrm{s})$ \\
\hline MAC & 202.11 \\
\hline Bandwidth & 32 \\
\hline CWmin & 1024 \\
\hline CWmax & $20 \mathrm{us}$ \\
\hline Slot time &
\end{tabular}

\section{PERFormance RESUlts}

Figure 9 depicts the average end-to-end delay (EED) of AODV, RDT-AODV, RMMMC and RIVC-MB protocols against the node density in the network. EED is the average time between transmitting a data packet at the source node and its successful reception at the destination node which measured in seconds. The Multichannel routing protocols achieve less EED compared with that in a single channel routing protocol. This is due to the availability of multiple orthogonal channels for communication. However, the EED in RDT-AODV increases significantly compared with the RMMMC and RIVC$\mathrm{MB}$ as the node density increases. This is because multiple paths are available to recover from a broken link in RMMMC and RIVC-MB. Although RMMMC and RIVC-MB use the same route discovery process, RIVC-MB achieves less EED as it relies only on a route with valid route information to recover from a broken link and also provides an early switchover if the route at the receiver node is invalid. Moreover, it also reduces the waiting time for a node which is attempting to access a busy medium by enabling an early switchover when the CW reached a pre-defined threshold.

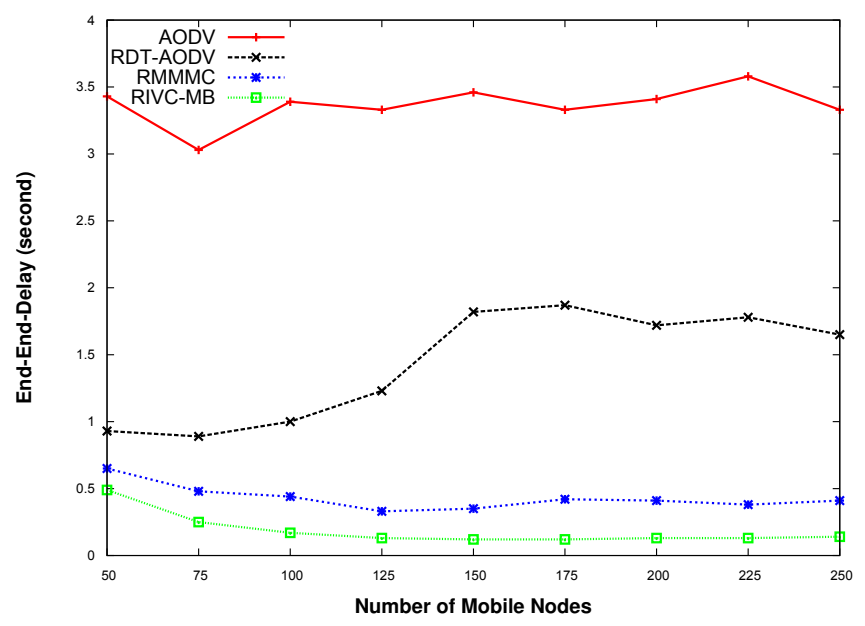

Fig. 9: End-to-End Delay.

Figure 10 shows the impact of node density on the performance of AODV, RDT-AODV, RMMMC and RIVC-MB proto- 
cols in terms of dropped data packets at the routing layer. They are caused by link breakage or the invalidity/unavailability of a route toward the destination at the receiver node. The multipath routing protocols have fewer dropped data packets compared with AODV and RDT-AODV protocols. This may be due to the availability of an alternative path when link breakage is detected. As is expected, RIVC-MB reduces the number of the dropped data packets at the routing layer significantly by about $159 \%$ compare with RMMMC protocol. This may be due to early invalid route detection and an early switchover process. RIVC-MB has a steady performance despite the increase of the network density with the exception of a low-density network which may be due to low connectivity in the network.

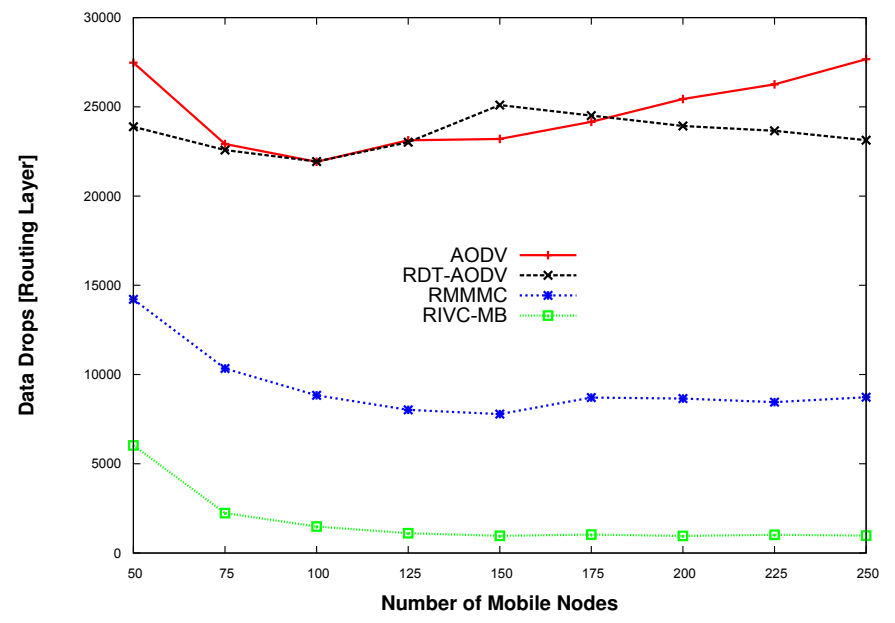

Fig. 10: Data packets dropped at the routing layer.

Figure 11 shows packet collisions rate at the MAC layer for the AODV, RDT-AODV, RMMMC and RIVC-MB protocols against node density in the network. It displays the number of dropped packets at the MAC layer caused by collisions per second. Figure 11 shows that the number of packet collisions at the MAC layer in AODV dramatically increases as the number of nodes increase. This is due to higher contending/collisions in a single shared medium compared with multiple shared mediums. Although RMMMC and RIVC-MB initiate two route discovery processes to any destination, they have fewer packet collisions in the MAC layer compared with RDTAODV. This is because RMMMC and RIVC-MB can recover from a broken link by using the available alternative route without initiating a new route discovery process. The proposed protocol has reduced the rate of packet collision at MAC layer by about $42 \%$ compared with RMMMC as it only enables data packets with valid route information to be transmitted/rererouted in the medium. Furthermore, it reduces the amount of contention/collisions in a busy medium by re-routing data packets via a valid alternative route, if available. These factors help to reduce the number of the data packets with invalid routing information that clog the medium. Moreover, it reduces contention in a busy medium, which helps to reduce the number of collisions in the medium.

Figure 12 shows the number of Route ERRor control packet (RERR) at the routing layer for the AODV, RDT-AODV,

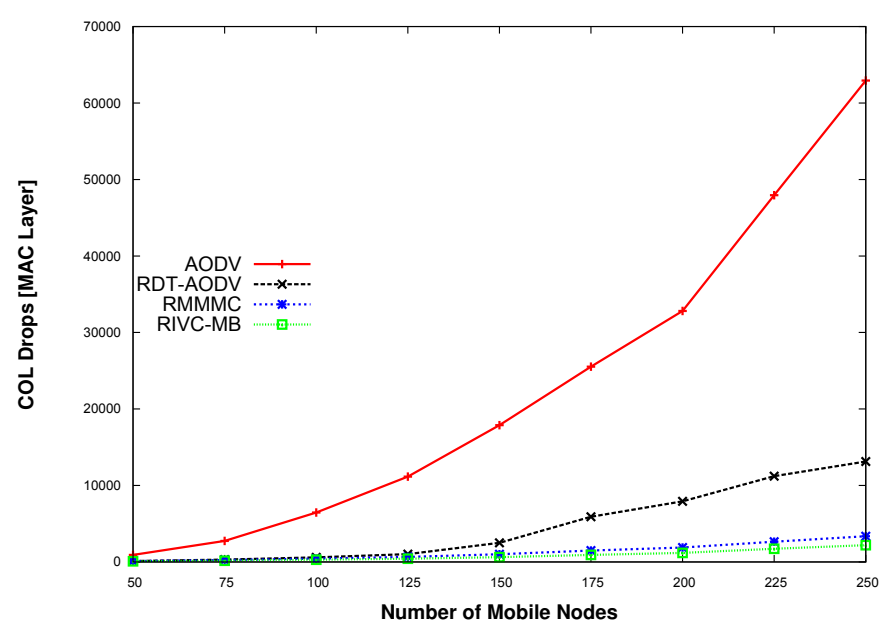

Fig. 11: Collided packet at MAC layer.

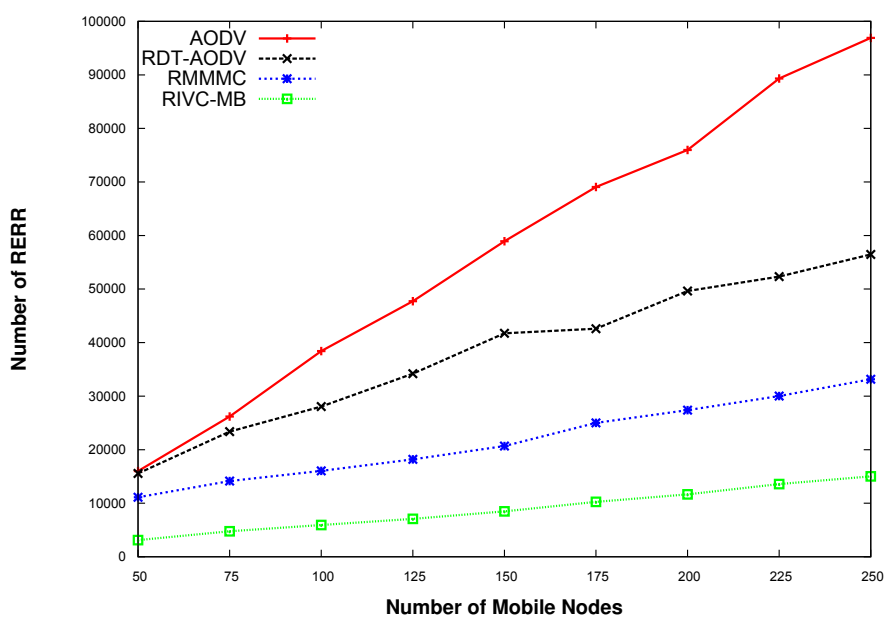

Fig. 12: Route Error packet at routing layer.

RMMMC and RIVC-MB protocols against node density in the network. A RERR packet is sent in the following situation: if the node detects a link breakage and node cannot repair it, receives a data packet to forward while it has invalid route information and receives RERR from a neighbour for one or more active routes in its routing table. Figure 12 shows that the number of RERR packets at the routing layer in AODV is the highest among the other protocols. This is because the number of link breakages in a single shared medium is higher than that in multiple shared mediums. The multipath multichannel routing protocols issue fewer RERR packets compared with the single path multichannel RDT-AODV protocol. This is because RMMMC and RIVC-MB can recover from a broken link by using the available alternative route. However, as RMMMC may rely on an alternative route with an invalid route to recover from the broken link, it has a higher number of sent RERR compared with RIVC-MB. The proposed protocol has reduced the number of sent RERR by about $75 \%$ compared 
with the RMMMC. This is because it provides an early route invalidity detection and an early switchover to the alternative route, which mitigates the occurrence of dropped data packets due to the invalid route. Furthermore, because the proposed protocol reduces the amount of contention/collisions in a busy medium by re-routing the data packets to the alternative route when the retransmission threshold reached.

\section{CONCLUSIONS AND FUTURE WORK}

In this paper, we have presented a new MAC protocol called (RIVC-MB) to improve the reliability in transmitting/rerouting in a multipath multichannel wireless ad-hoc network. RIVC-MB protocol utilises cross-layer interaction with the routing layer to add two enhancement mechanisms to a multipath multichannel network. The first mechanism is Route Information Validity Check (RIVC) which mitigates the issue of transmitting/re-routing a data packet to a node which has invalid route information towards the final destination. It provides early route invalidity detection and early switchover. The second mechanism is Modified Backoff (MB) which reduces the waiting time for a node which is attempting to access a busy medium. Using an NS2 simulation, we compared the performance of the proposed protocol with the standard AODV, RDT-AODV and RMMMC under different network densities. The simulation results showed that the RIVC-MB protocol has significantly reduced the end-to-end delay of data packets. Also, it reduced the number of dropped data packets and the number of sent route error packets at the routing layer. In addition, it reduced the number of packet collisions in the MAC layer.

In our future work, we intend to evaluate RIVC-MB under different network contexts (high load, high mobility and single channel network).

\section{REFERENCES}

[1] Z. Iqbal, S. Khan, A. Mehmood, J. Lloret, and N. A. Alrajeh, "Adaptive cross-layer multipath routing protocol for mobile ad hoc networks," Journal of Sensors, vol. 2016, 2016.

[2] N. Soni and S. S. Tripathi, "Multipath routing protocol for wireless sensor networks," 2017.

[3] K. Wu and J. Harms, "Performance study of a multipath routing method for wireless mobile ad hoc networks," in MASCOTS 2001, Proceedings Ninth International Symposium on Modeling, Analysis and Simulation of Computer and Telecommunication Systems, 2001, pp. 99-107.

[4] M. R. Pearlman, Z. J. Haas, P. Sholander, and S. S. Tabrizi, "On the impact of alternate path routing for load balancing in mobile ad hoc networks," in 2000 First Annual Workshop on Mobile and Ad Hoc Networking and Computing. MobiHOC (Cat. No.OOEX444), 2000, pp $3-10$.

[5] I. L. M. S. Committee et al., "Wireless lan medium access control (mac) and physical layer (phy) specifications," IEEE Std, vol. 802, 2007.

[6] Z. Chen, R. D. Yates, and D. Raychaudhuri, "Dynamic node-disjoint multipath routing for millimeter wave networks using directional antennas," in Information Science and Systems (CISS), 2016 Annual Conference on. IEEE, 2016, pp. 430-435.

[7] C. Pan, B. Liu, H. Zhou, and L. Gui, "Multi-path routing for video streaming in multi-radio multi-channel wireless mesh networks," in Communications (ICC), 2016 IEEE International Conference on. IEEE, 2016, pp. 1-6.

[8] N. S. Mirza, M. A. Abdelshafy, and P. J. King, "Performance evaluation of receiver directed transmission protocol with a single transceiver in manets," in Wireless Days, 2017. IEEE, 2017, pp. 241-244.
[9] N. S. Mirza, P. J. B. King, I. Romdhani, M. A. Abdelshafy, and A. A. Alghamdi, "Reliable multipath multi-channel route migration over multi link-failure in wireless ad hoc networks," in 2017 IEEE 13th International Conference on Wireless and Mobile Computing, Networking and Communications (WiMob), Oct 2017, pp. 123-130.

[10] C. E.-A. Campbell, K.-K. Loo, O. Gemikonakli, S. Khan, and D. Singh, "Multi-channel distributed coordinated function over single radio in wireless sensor networks," Sensors, vol. 11, no. 1, pp. 964-991, 2011.

[11] N. Shacham and P. King, "Architectures and performance of multichannel multihop packet radio networks," IEEE Journal on Selected Areas in Communications, vol. 5, no. 6, pp. 1013-1025, 1987.

[12] "The Network Simulator NS-2, http://www.isi.edu/nsnam/ns/ [retrieved: January, 2018]."

[13] K. Fall and K. Varadhan, "The network simulator ns-2: Documentation," 2007.

[14] C. Perkins, E. Belding-Royer, and S. Das, "Ad hoc on-demand distance vector (aodv) routing,” Tech. Rep., 2003. 